\title{
Acute Isolated Dysarthria Is Associated with a High Risk of Stroke
}

\author{
Alina Beliavsky c Jeffrey J. Perry a, d Dar Dowlatshahi b-d \\ Jason Wasserman c Marco L.A. Sivilotti e, $f$ Jane Sutherland d \\ Andrew Worster j Marcel Émond h Grant Stotts c Albert Y. Jing \\ Wieslaw J. Oczkowski k Demetrios J. Sahlas k Heather E. Murray e \\ Ariane MacKey i Steve Verreault i George A. Wells b Ian G. Stiell a, c, d \\ Mukul Sharma d, k
}

Departments of a Emergency Medicine and b Epidemiology and Community Medicine, and c Division of Neurology, University of Ottawa, and d Clinical Epidemiology Program, Ottawa Hospital Research Institute, Ottawa, Ont., Departments of e Emergency Medicine and f Biomedical and Molecular Sciences, and g Division of Neurology, Queen's University, Kingston, Ont., h Department of Emergency and Family Medicine, Université Laval, and i Department of Neurology, Hôpital de I'Enfant-Jésus, Quebec City, Quebec, Que., and Divisions of j Emergency Medicine and k Neurology, McMaster University, Hamilton, Ont., Canada

Key Words

Stroke · Transient ischemic attack · Risk stratification · Outcome · Dysarthria

\section{Abstract}

Background: Isolated dysarthria is an uncommon presentation of transient ischemic attack (TIA)/minor stroke and has a broad differential diagnosis. There is little information in the literature about how often this presentation is confirmed to be a TIA/stroke, and therefore there is debate about the risk of subsequent vascular events. Given the uncertain prognosis, it is unclear how to best manage patients presenting to the emergency department (ED) with isolated dysarthria. The objective of this study was to prospectively identify and follow a cohort of patients presenting to EDs with isolated dysarthria in order to explore their natural history and risk of recurrent cerebrovascular events. Specifically, we sought to determine early outcomes of individuals with this nonspecific and atypical presentation in order to appropriately expedite their management. Methods: Patients with isolated dysarthria having presented to 8 Canadian EDs between October 2006 and April 2009 were analyzed as part of a prospective multicenter cohort study of patients with acute neurological symptoms as assessed by emergency physicians. The study inclusion criteria were age $\geq 18$ years, a normal level of consciousness, and a symptom onset $<1$ week prior to presentation without an established nonvascular 
etiology. The primary outcome was a subsequent stroke within 90 days of the index visit. The secondary outcomes were the rate of TIA, myocardial infarction, and death. Isolated dysarthria was defined as slurring with imprecise articulation but without evidence of language dysfunction. The overall rate of stroke in this cohort was compared with that predicted by the median ABCD2 score for this group. Results: Between 2006 and 2009, 1,528 patients were enrolled and had a 90-day follow-up. Of these, 43 patients presented with isolated acuteonset dysarthria (2.8\%). Recurrent stroke occurred in 6/43 (14.0\%) within 90 days of enrollment. The predicted maximal 90-day stroke rate was $9.8 \%$ (based on a median ABCD2 score of 5 for the isolated dysarthria cohort). After adjusting for covariates, isolated dysarthria independently predicted stroke within 90 days (aOR: 3.96; 95\% CI: 1.3-11.9; $p=0.014$ ). Conclusions: The isolated dysarthria cohort carried a recurrent stroke risk comparable to that predicted by the median $A B C D 2$ scores. Although isolated dysarthria is a nonspecific and uncommon clinical presentation of TIA, these findings support the need to view it first and foremost as a vascular presentation until proven otherwise and to manage such patients as if they were at high risk of stroke in accordance with established high-risk TIA guidelines.

(C) 2014 S. Karger AG, Basel

\section{Introduction}

Isolated acute-onset dysarthria is an uncommon presentation of transient ischemic attack (TIA)/minor stroke in the emergency department (ED); published case series estimate its occurrence in $<1.3 \%$ of confirmed strokes $[1,2]$. The differential diagnosis for this clinical presentation is broad and ranges from cranial neuropathies [3] and acute metabolic disturbances [4] to inflammatory diseases [5]. It has an unknown prognosis, and there have been no published reports describing its natural history. The objectives of this study were to prospectively identify and follow a cohort of patients presenting to EDs with acute isolated dysarthria on physical examination and to establish their 90-day rates of stroke, recurrent TIA, myocardial infarction (MI), and all-cause mortality.

\section{Methods}

The data for this study were collected from a previously described prospective multicenter study [6]. It identified and followed a cohort of patients presenting with acute focal neurological findings compatible with TIA (as per emergency physician assessment) to 8 Canadian academic EDs between October 2006 and April 2009. The Ottawa Hospital Research Ethics Boards (OHREB) approved the study. Due to the observational nature of the study, we obtained a waiver of consent from the OHREB for enrollment, but verbal consent was obtained to complete the telephone follow-up (also approved by the OHREB). The inclusion criteria were age $\geq 18$ years and an emergency physician diagnosis of TIA/minor stroke. Exclusion criteria were an onset $>1$ week prior to presentation, a decreased level of consciousness, or an established nonvascular etiology for the symptoms.

Additional criteria for this substudy were the presence of dysarthria on examination with the absence of any weakness, pronator drift, diplopia, visual field defect, language deficit, altered sensation, abnormal finger-to-nose test results, or gait abnormality. Dysarthria was defined as slurring with imprecise articulation but without evidence of language dysfunction [2]. The primary outcome was stroke at 90 days. The secondary outcomes were the rate of TIA, MI [7], and death. As an exploratory outcome, we compared the overall rate of stroke with that predicted by the median ABCD2 score [8] for the isolated dysarthria cohort. The 
outcomes were assessed by clinical follow-up, chart review, and/or a validated standardized telephone interview [9] at 7 and 90 days, and all patients with a possible subsequent event were adjudicated by a three-physician committee (consisting of at least one stroke neurologist); the outcome adjudication was blinded to the original data collection forms $[6,10]$.

To determine if isolated dysarthria independently predicted stroke at 90 days, we built a backward stepwise logistic regression model including covariates identified from exploratory univariate analysis with $\mathrm{p}<0.10$. We compared the stroke rate in the isolated dysarthria group with the rate predicted by ABCD2 scoring, using a two-sided Fisher exact test.

\section{Results}

Between 2006 and 2009, 1,784 patients were enrolled in the study, of which 1,528 had 90-day follow-up data. In this cohort, 43 patients (2.8\%) had isolated dysarthria on examination; their median age was 78 years (IQR: $67-89$ ), and $60.5 \%$ were female. Of these patients with isolated dysarthria, $6(14.0 \%)$ had a recurrent stroke within 90 days.

Three strokes occurred within 2 days, 1 within 7 days, 1 within 30 days, and the last within 90 days. Univariate analyses identified age, hypertension, coronary artery disease, carotid stenosis, ASA use at the time of the index event, and ABCD2 score as covariates for the regression model. After logistic regression, only isolated dysarthria (aOR: 3.96; 95\% CI: 1.3-11.9; $\mathrm{p}=0.014$ ) and ABCD2 score (OR: 1.37 per point; $95 \%$ CI: $1.1-1.7 ; \mathrm{p}=0.005$ ) predicted 90-day stroke. The predicted maximal 90-day stroke rate was $9.8 \%$ (based on a median ABCD2 score of 5 for the isolated dysarthria cohort). Although the absolute 90-day stroke rate in the isolated dysarthria cohort was higher than the predicted rate, this was not statistically significant $(p=0.74)$. In the isolated dysarthria group, there were 1 TIA and 2 deaths but no MI within 90 days.

\section{Discussion}

Since acute-onset isolated dysarthria is an uncommon clinical presentation of TIA, it may be perceived as a nonspecific neurological syndrome with a broad differential diagnosis [11, 12]. We present prospective observational data demonstrating a 14.0\% 90-day stroke risk, suggesting that isolated dysarthria is indeed a high-risk vascular presentation.

The isolated dysarthria cohort carried a recurrent stroke risk comparable to that predicted by the median ABCD2 scores. While this does not necessarily imply that the clinical phenotype of isolated dysarthria should be used as a new risk stratification tool above the existing predictive scores, it does highlight the need to view it first and foremost as a vascular presentation until proven otherwise. Management of this clinical entity should be expedited in accordance with established high-risk TIA guidelines [13].

A limitation of this study is the small sample size of patients presenting with isolated dysarthria (43), with only 6 recurrent stroke events in this group. This can lead to imprecision in the estimation of risk for this item, which might relate to the high OR obtained (3.96). However, the significantly elevated 90-day recurrent stroke risk for patients presenting with this symptom still warrants attending to the finding that isolated dysarthria is a high-risk presentation in ischemic stroke.

Our results are observational and should be interpreted with caution. Although a threephysician panel adjudicated the outcomes, there was no magnetic resonance imaging requirement to confirm the diagnosis of stroke, to establish the etiology of the index or recurrent stroke, or to differentiate a recurrent stroke from the progression of the same 
stroke. Further, we were not able to determine the effect of any medical interventions initiated in the EDs on our outcomes, although all the patients in the study had antiplatelet therapy initiated. In addition, since this was a pragmatic study where nonneurologists assessed and enrolled the patients, the pattern, severity, or type of dysarthria in the presenting patients was not clearly documented. Finally, the initial decision to enroll patients in the study was made based on the assessment of an emergency physician, which may differ from an assessment made by a consultant neurologist.

Nevertheless, our data are applicable to patients seen by emergency physicians who must determine the most appropriate initial management and referral pathway as well as the urgency of this referral. In this context, our data suggest patients presenting with acute isolated dysarthria be managed as if they were at high risk of stroke.

\section{References}

1 Arboix A, López-Grau M, Casasnovas C, García-Eroles L, Massons J, Balcells M: Clinical study of 39 patients with atypical lacunar syndrome. J Neurol Neurosurg Psychiatry 2006;77:381-384.

$\checkmark 2$ Kumral E, Celebisoy M, Celebisoy N, Canbaz DH, Calli C: Dysarthria due to supratentorial and infratentorial ischemic stroke: a diffusion-weighted imaging study. Cerebrovasc Dis 2007;23:331-338.

3 Graham RM, Thomson EF, Baldwin AJ: Isolated hypoglossal nerve palsy due to a vascular anomaly. Int J Oral Maxillofac Surg 2007;36:759-761.

4 Manto MU: Isolated cerebellar dysarthria associated with a heat stroke. Clin Neurol Neurosurg 1996;98: 55-56.

5 Ramesha KN, Rajesh B, Ashalatha R, Kesavadas C, Abraham M, Radharkrishnan VV, Sarma PS, Radharkrishnan K: Rasmussen's encephalitis: experience from a developing country based on a group of medically and surgically treated patients. Seizure 1996;18:567-572.

6 Wasserman J, Perry J, Dowlatshahi D, Stotts G, Stiell I, Sutherland J, Symington C, Sharma M: Stratified, urgent care for transient ischemic attack results in low stroke rates. Stroke 2010;41:2601-2605.

7 Gattringer T, Niederkorn K, Seyfang L, Seifert-Held T, Simmet N, Ferrari J, Lang W, Brainin M, Willeit J, Fazekas F, Enzinger C: Myocardial infarction as a complication in acute stroke: results from the Austrian Stroke Unit Registry. Cerebrovasc Dis 2014;37:147-152.

8 Rothwell PM, Giles MF, Flossmann E, Lovelock CE, Redgrave JN, Warlow CP, Mehta Z: A simple score (ABCD) to identify individuals at high early risk of stroke after transient ischemic attack. Lancet 2005;366:29-36.

9 Meschia JF, Brott TG, Chukwudelunzu FE, et al: Verifying the stroke-free phenotype by structured telephone interview. Stroke 2000;31:1076-1080.

10 Perry JJ, Sharma M, Sivilotti ML, Sutherland J, Symington C, Worster A, Émond M, Stotts G, Jin AY, Oczkowski WJ, Sahlas DJ, Murray HE, MacKey A, Verreault S, Wells GA, Stiell IG: Prospective validation of the ABCD2 score for patients in the emergency department with transient ischemic attack. CMAJ 2011;183:1137-1145.

11 Amort M, Fluri F, Schäfer J, Weisskopf F, Katan M, Burow A, Bucher HC, Bonati LH, Lyrer PA, Engelter ST: Transient ischemic attack versus transient ischemic attack mimics: frequency, clinical characteristics and outcome. Cerebrovasc Dis 2011;32:57-64.

12 Prabhakaran S, Silver AJ, Warrior L, McClenathan B, Lee VH: Misdiagnosis of transient ischemic attacks in the emergency room. Cerebrovasc Dis 2008;26:630-635.

13 Furie KL, Kasner SE, Adams RJ, Albers GW, Bush RL, Fagan SC, Halperin JL, Johnston SC, Katzan I, Kernan WN, Mitchell PH, Ovbiagele B, Palesch YY, Sacco RL, Schwamm LH, Wassertheil-Smoller S, Turan TN, Wentworth D: Guidelines for the prevention of stroke in patients with stroke or transient ischemic attack: a guideline for healthcare professionals from the American Heart Association/American Stroke Association. Stroke 2011;42: 227-276. 